\title{
SHARAQ Spectrometer: High-resolution Spectroscopy Using Exotic Beams and Reactions
}

\author{
S. Michimasa*, ${ }^{a}$ S. Ota,${ }^{a}$ M. Dozono, ${ }^{a}$ M. Takaki, ${ }^{a}$ K. Kisamori, ${ }^{a}$ H. Miya, ${ }^{a}$ \\ M. Kobayashi, ${ }^{a}$ Y. Kiyokawa, ${ }^{a}$ H. Baba, ${ }^{b}$ N. Fukuda, ${ }^{b}$ N. Inabe, ${ }^{b}$ S. Kawase, ${ }^{c}$ \\ T. Kubo, ${ }^{b}$ Y. Kubota, ${ }^{b}$ C.S. Lee,${ }^{a}$ M. Matsushita, ${ }^{a}$ H. Sakai, ${ }^{b}$ A. Stolz,${ }^{d}$ H. Tokieda, ${ }^{a}$ \\ T. Uesaka, ${ }^{b}$ K Yako, ${ }^{a}$ Y. Yanagisawa, ${ }^{b}$ R. Yokoyama, ${ }^{a}$ K Yoshida ${ }^{b}$ and S. Shimoura ${ }^{a}$ \\ ${ }^{a}$ Center for Nuclear Study, the University of Tokyo, 2-1 Hirosawa, Wako, Saitama 351-0198, \\ Japan \\ ${ }^{b}$ RIKEN Nishina Center , 2-1 Hirosawa, Wako, Saitama 351-0198, Japan \\ ${ }^{c}$ Department of Advanced Energy Engineering Sciences, Kyushu University, 6-1 Kasuga-koen, \\ Kasuga, Fukuoka 816-8560, Japan \\ ${ }^{d}$ National Superconducting Cyclotron Laboratory, Michigan State University, 640 S Shaw Ln, \\ East Lansing, MI 48824, U.S.A. \\ E-mail: mitimasadens.s.u-tokyo.ac.jp
}

\begin{abstract}
We introduce the recent development for increasing the performance of the SHARAQ spectrometer, which was operated at RI Beam Factory in RIKEN, Japan. The paper contains main achievements of our developments, which are detector developments, improvements of high-resolution performance at SHARAQ, and developments of new reaction probes to explore new kinds of nuclear responses. By using those new experimental technique, we recently performed various nuclear experiments at SHARAQ. We described here the preliminary results of the experiments together with their brief details.
\end{abstract}

The 26th International Nuclear Physics Conference

11-16 September, 2016

Adelaide, Australia

\footnotetext{
* Speaker.
} 


\section{Introduction}

The SHARAQ ${ }^{1}$ spectrometer [U, [1] and High-Resolution Beamline [3] (HRB), which began operation in March 2009 at the RI beam Factory (RIBF), were constructed for aiming at highresolution spectroscopy with reactions induced by radioactive ions (RI's) at $100 \mathrm{~A}-350 \mathrm{~A} \mathrm{MeV}$ using the missing-mass method and exploring new experimental techniques in the field of nuclear physics. The HRB is located at the downstream of BigRIPS [四], where RI beams are produced by fragmentation and/or fission reactions from a primary nuclear beam such as ${ }^{238} \mathrm{U}$. The momentumdispersive focal planes with large dispersion are set in the middle of HRB and they are useful for measurements of beam particle momenta on an event-by-event basis. The SHARAQ spectrometer is a high-resolution magnetic analyzer consisting of two superconducting quadrupoles, one normal conducting quadrupole and two dipole magnets in a "QQDQD" configuration. For missing mass measurements with extremely good resolution, the SHARAQ and HRB are designed to achieve both lateral and angular dispersion matching. Figure $\square$ shows the schematic view of BigRIPS, HRB and SHARAQ. The detectors in this figure were used in the direct mass measurement[可],

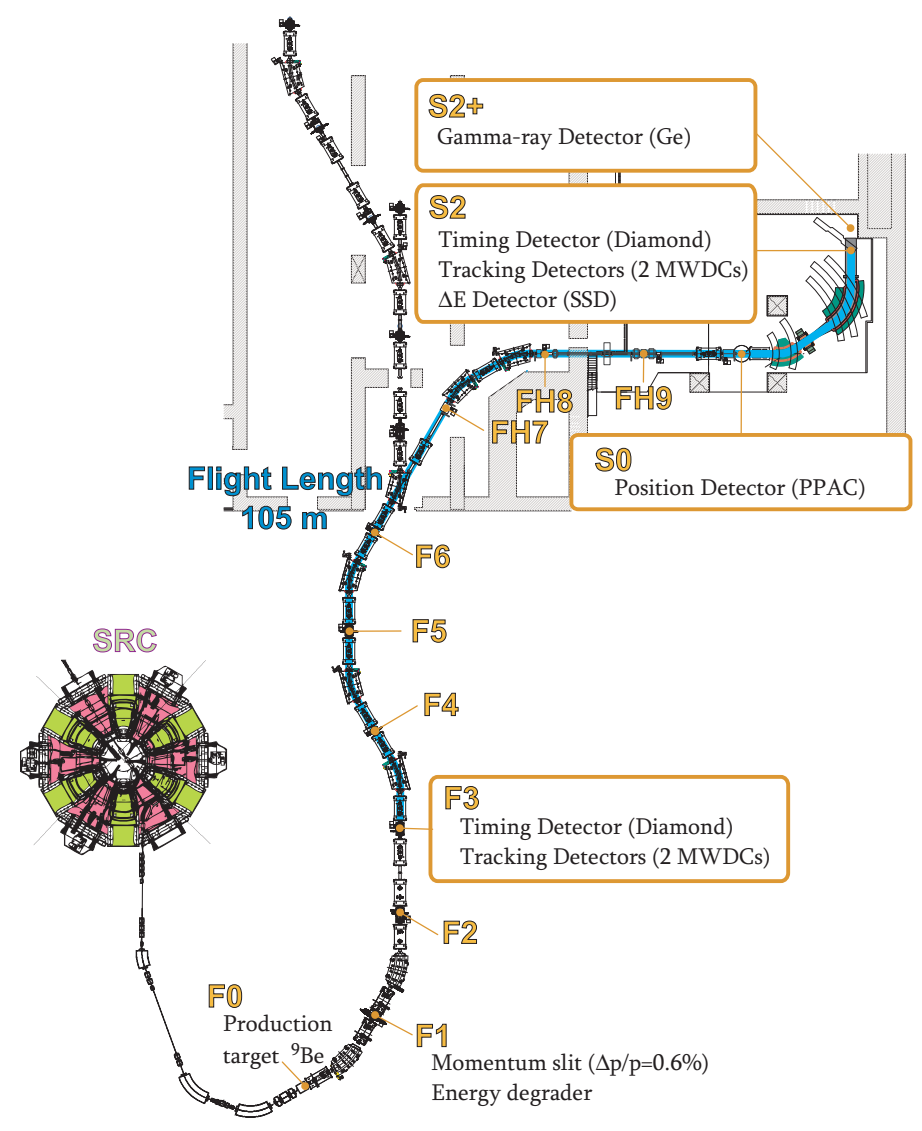

Figure 1: Schematic view of the BigRIPS, the High-Resolution Beamline and the SHARAQ spectrometer. The detector setup shown in this figure was for the direct mass measurement[[]].

described details later.

\footnotetext{
${ }^{1}$ Spectrocopy of High-resolution Analyzer of RadioActive Quantum beams
} 
RI's that possess large isospin degrees of freedom are very interested in their stability, such as the masses and the nuclear structures. Additionally reactions with RI's are also expected to open new research opportunities in nuclear excitation studies. SHARAQ pursues nuclear studies on the structures and responses of RI's with high-resolution nuclear spectroscopy. This paper describes technical developments for improving the performance of SHARAQ with outlines of recently performed experiments.

\section{Development of Detectors}

For physics measurements with high resolution, the SHARAQ system must be set to satisfy the designed ion-optical condition for each experiment. As the standard setup, we install tracking detectors at the F3, F4, F5, F6, FH7, FH9, S0 and S2 focal planes and construct the designed ionoptics for experiments in the sequential order. The ion-optical condition between the two foci is checked by using the correlations of beam profiles at those foci. We finally set the system to satisfy the designed transport matrix elements, such as the momentum dispersion, focusings and magnifications, of the beamline and spectrometer. For the tracking detectors for SHARAQ, we adopted multi-wire drift chambers, operated at low gas pressure (LP-MWDC's) [目]. This LP-MWDC enables us the compatibility of high beam rate capability and high position resolution, even for light particles. As typical values, the LP-MWDC performed the position resolution of better than 300 $\mu \mathrm{m}$ (FWHM) under more intense RI beams than 1 Mcps during 1-week experiment. The tracking detectors are used both for diagnosis of the ion transport optics and for event-by-event tagging of particles in experiments at SHARAQ. The SHARAQ collaboration continues developments of standard detectors in order to optimize their performance.

\subsection{Isomer Tagging System for SHARAQ}

When high-resolution nuclear spectroscopy applies to heavier RI regions, the confirmation of particle identification (PID) is critical for tagging the reaction channel. Generally, heavier RI beam produced by using an in-flight separator, such as BigRIPS, includes several nuclei at the same time. Therefore, the above-mentioned ion-optical tuning must be performed after the PID was confirmed. For an extension of SHARAQ performance, we newly installed an isomer tagging system at S2+, where is the downstream of the final focal plane of SHARAQ (S2). The details

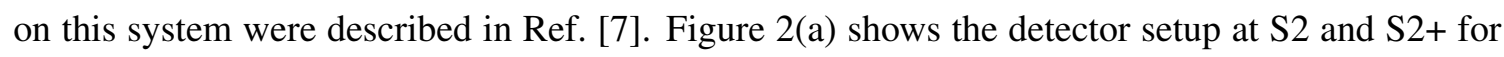
heavier RI beams. The S2 Diamond and S2 SSD were used to obtain time of flight (TOF) and $\Delta \mathrm{E}$, respectively. Particle identification was achieved by TOF- $\Delta$ E-B $\rho$ method [ [8]]. Figure $\mathbb{D}(\mathrm{b})$ shows the online performance of PID. We fixed the ion-optical setting using this PID information. At S2+, 3 plastic scintillators and $\gamma$-ray detectors were installed for measuring delayed $\gamma$-rays from isomer states. The beam particles passed through a $\mathrm{S} 2+\mathrm{Al}$ degrader degrading their energy and stopped at $\mathrm{S} 2+$ plastic stopper. $\mathrm{S} 2+$ plastic veto was installed for detecting events that fragmentation reactions occur in the degrader or the stopper. The $\gamma$-ray detectors consisted in 2 Ge detectors and $16 \mathrm{NaI}(\mathrm{Tl})$ scintillators. The delayed $\gamma$-ray data were restored combined with event-by-event PID information by using time-stamping system [Q]].

The isomer tagging system reconfirmed known isomers which is shown by orange circles in Fig. $\square($ (b). Therefore, the $Z$ and $A / Q$ axes of this figure were completely calibrated. Additionally 
(a) Detector setup at S2+

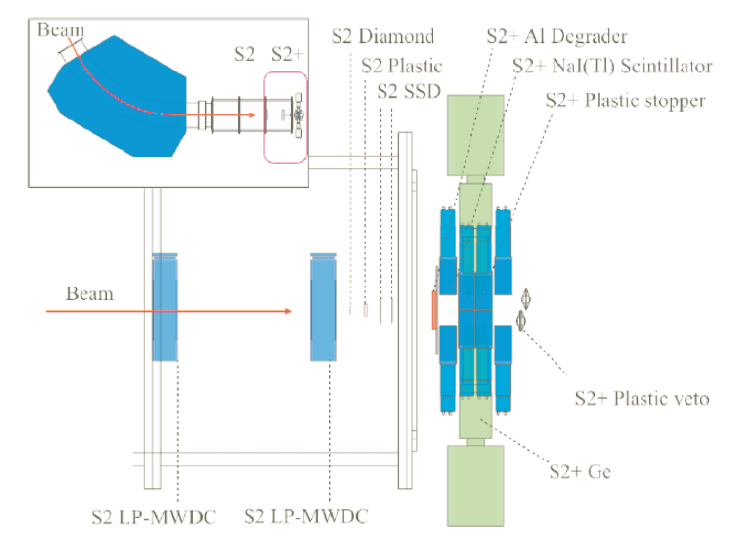

(b) Particle identification

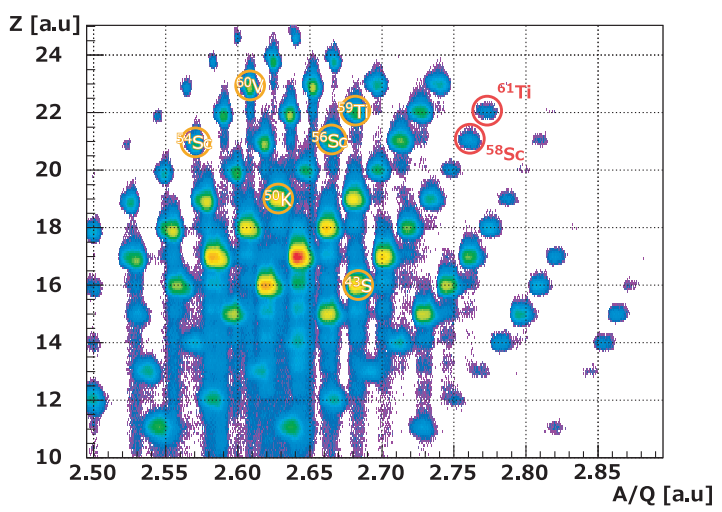

Figure 2: (a) Detector setup installed at S2 and S2+. (b) Particle identification on event-by-event basis

by using this system, the isomeric states at ${ }^{61} \mathrm{Ti}$ and ${ }^{58} \mathrm{Sc}$ were newly found, as shown in Fig. B]. We measured the $125.2-\mathrm{keV}$ and $575.1-\mathrm{keV} \gamma$ rays in ${ }^{61} \mathrm{Ti}$, and the $180.5-\mathrm{keV}, 247-\mathrm{keV}, 412.3-\mathrm{keV}$ and 580.9-keV $\gamma$ rays in ${ }^{58} \mathrm{Sc}$, for the first time. We are ongoingly checking whether these $\gamma$-rays
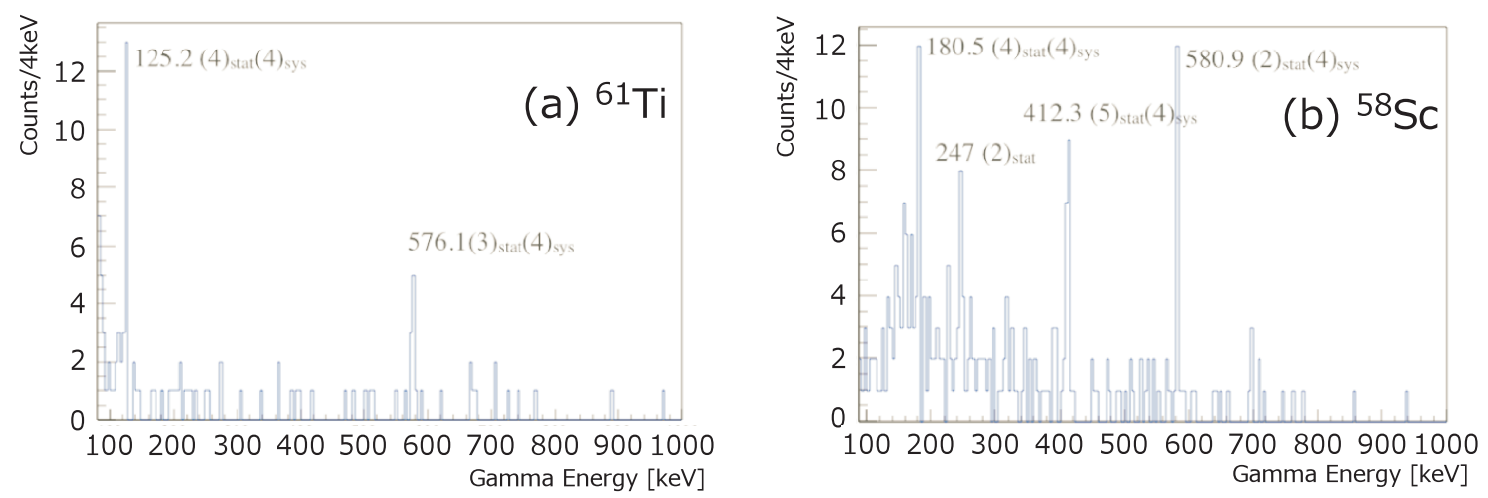

Figure 3: Delayed- $\gamma$ spectra from isomers in (a) ${ }^{61} \mathrm{Ti}$ and (b) ${ }^{58} \mathrm{Sc}$.

are $\gamma$ cascades or not.

\subsection{Diamond Detectors}

To improve the time resolution of time-of-flight measurements along the beamline, we developed diamond detectors in a collaboration with NSCL/MSU. The diamond detector consists of a policrystalline CVD diamond crystal with an active area of $28 \mathrm{~mm}^{2}$ and a thickness of $0.2 \mathrm{~mm}$, and has one pad on the other side (Side A) and four strips on one side (Side B). On the Side A, 4 readout wires are bonded to the corners. On the Side B, 2 readout wires are bonded to the both edges of each strip. The width of the four strips are 9, 5, 5, and $9 \mathrm{~mm}$ from the top. Details on the structure of CNS diamond detector were reported in Ref. [U]]. Figure $\mathbf{t}$ shows photographs and cathode patterns of the CNS diamond detector.

By using the irradiation data on many nuclei shown in Fig. $\square(b)$, we evaluated the energyloss dependence of the timing resolution of the diamond detector. The evaluation procedure of 

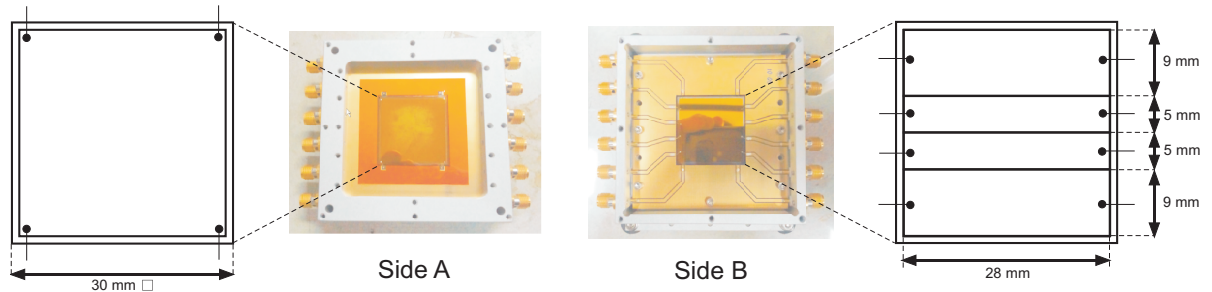

Figure 4: Photographs and cathode pattern of the CNS diamond detector.

time resolution for each nucleus was the same with that in Ref. [ए]]. Agilent TC842 and optical signal transfer system were used as the data acquisition system. The hit-position dependence of the time difference was compensated by using the position information deduced from the tracking detectors. For ${ }^{57} \mathrm{Sc}$, the standard deviation of the time difference was evaluated to be $17 \mathrm{ps}(\sigma)$ from the spectrum shown in Fig. B(a). Consequently, the difference value indicates that the time
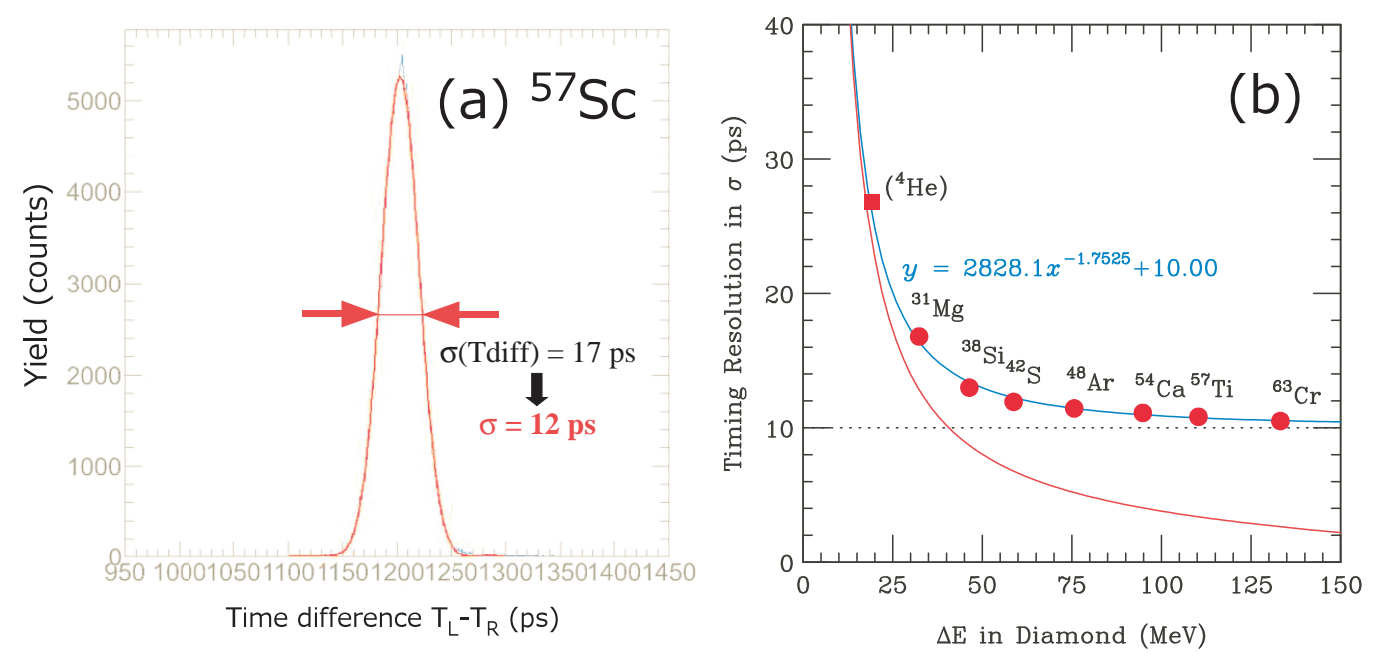

Figure 5: (a) Time-difference spectrum for ${ }^{57} \mathrm{Sc}$. The difference is calculated from the both sides of the strip. (b) Time resolution of the CVD diamond detector as a function of the energy loss. The circle (square) points show the obtained values for isotopes in the present (previous[[0]) experiment. The blue curve is obtained by fitting to the data. The lower curve shows intrinsic time resolution of a diamond detector when the time resolutions of the optical fiber is $10.2 \mathrm{ps}(\sigma)$.

resolution of the diamond is $12 \mathrm{ps}(\sigma)$ for this nucleus. The time resolutions for other nuclei were also obtained by the same procedure. Figure 5(b) shows the deduced time resolutions as a function of the energy deposit in the diamond detector. The data points in the figure show the obtained values from the measurements. The circles were obtained in the present experiment, while a square was already reported in Ref. [四].

The energy-loss dependence of the time resolution was well reproduced by a power function of the energy deposit with an offset, which is deduced as $\sigma_{\text {meas }}=2828.1(\Delta E)^{-1.7525}+10.00$ by a fitting. The offset component in the above function is considered to contain the time resolution of the data acquisition system. The resolution of the data acquisition system was able to be estimated from the data for time jitters with changing a transfer fiber length. Since the signal transfer fiber was 158 meters long in the experiment, we estimated the time resolution of the data acquisition 
system $\left(\sigma_{\mathrm{daq}}\right)$ to be $10.2 \mathrm{ps}(\sigma)$. This means that the offset of the energy-loss dependence was dominated by the resolution of the data acquisition system.

The intrinsic time resolution of the diamond detector can be evaluated by $\sigma_{\text {int }}=\sqrt{\sigma_{\text {meas }}^{2}-\sigma_{\text {daq }}^{2}}$, and its energy-loss dependence shows in Fig. 5(b). Finally, the intrinsic time resolution of the diamond detector is evaluated to be as good as $8 \mathrm{ps}(\sigma)$ for $50-\mathrm{MeV}$ deposit and $4 \mathrm{ps}(\sigma)$ for $100-\mathrm{MeV}$ deposit.

\section{Combine missing-mass spectroscopy with invariant mass technique}

The SHARAQ spectrometer is a sophisticated momentum analyzer for outgoing beam-like particles. As a novel reaction probe to explore a new aspect of nuclear response, we are developing the measurement technique for reactions where the outgoing particle is unbound nuclei in the normal kinematics with both radioactive and stable nuclear beams. In this aspect, we have examined the measurements of the $\left({ }^{8} \mathrm{He},{ }^{8} \mathrm{Be}\right)$ reaction []] and $\left({ }^{16} \mathrm{O},{ }^{16} \mathrm{~F}\right)$ reaction [ㅁ] ].

The $\left({ }^{8} \mathrm{He},{ }^{8} \mathrm{Be}\right)$ reaction was developed for aiming at producing a tetra-neutron state from ${ }^{4} \mathrm{He}$. This reaction kinematically match very much to the $4 \mathrm{n}$ state, because it is an exothermic doublecharge exchange (DCX) reaction. Another feature of this reaction is that outgoing particle immediately decays into $2 \alpha$ particles. Standard tracking detectors installed at the final focal plane of the SHARAQ spectrometer was Cathode-Readout Drift Chambers (CRDC's). For the capability of multi-particle detection, we adopted flash ADC's in the drift-time measurement of the CRDC's. Consequently two $\alpha$ particles was distinguished when their horizontal and vertical distances were $>10 \mathrm{~mm}$ and $>5 \mathrm{~mm}$, respectively, and thus the CRDC system with Flash ADC was able to identify $95 \%$ of the $\alpha$ pairs that decay from the ${ }^{8} \mathrm{Be}$ ground state. Further details on this experiment was reported in Ref. [ए]]. The $\left({ }^{16} \mathrm{O},{ }^{16} \mathrm{~F}\right)$ reaction was developed as a leading probe searching for the $0^{-}$excitation mode of nucleus. To measure the reaction, we developed a new ion-optical setting of SHARAQ spectrometer, called separation-flow mode [ए]]. The schematic setup for the separationflow mode is shown in Fig. 6 . The outgoing ${ }^{16} \mathrm{~F}$ is an unbound nucleus and suddenly decays into a ${ }^{15} \mathrm{O}$ nucleus and a proton. In this mode, Oxygen particles travel to the S2 focal plane, while protons are detected at the $\mathrm{S} 1$ focal plane. Therefore, two different kinds of outgoing particles are detected at the same time. Finally we successfully performed coincidence measurements of ${ }^{15} \mathrm{O}$ and a proton and the excitation energies of ${ }^{16} \mathrm{~F}$ 's were determined with $100 \mathrm{keV}$ (FWHM) from the invariant mass between an ${ }^{15} \mathrm{O}$ and a proton. Further details on this experiment was reported in Ref. [12]].

\section{Direct mass measurement for RI's far from the stability line.}

The nuclear masses are one of the most fundamental physics quantities for investigation of the nuclear stability between the Heisenberg valley to the dripline. Because of the high-momentum resolution of the BigRIPS-HRB-SHARAQ system and excellent TOF resolution provided by the pCVD diamond detectors, the performance of the system can achieve direct mass measurements with a sufficient accuracy to discuss on the systematics of the nuclear stability [[5]. The experimental setup is shown in Fig. . In the experiment, nuclear masses are determined by using the 


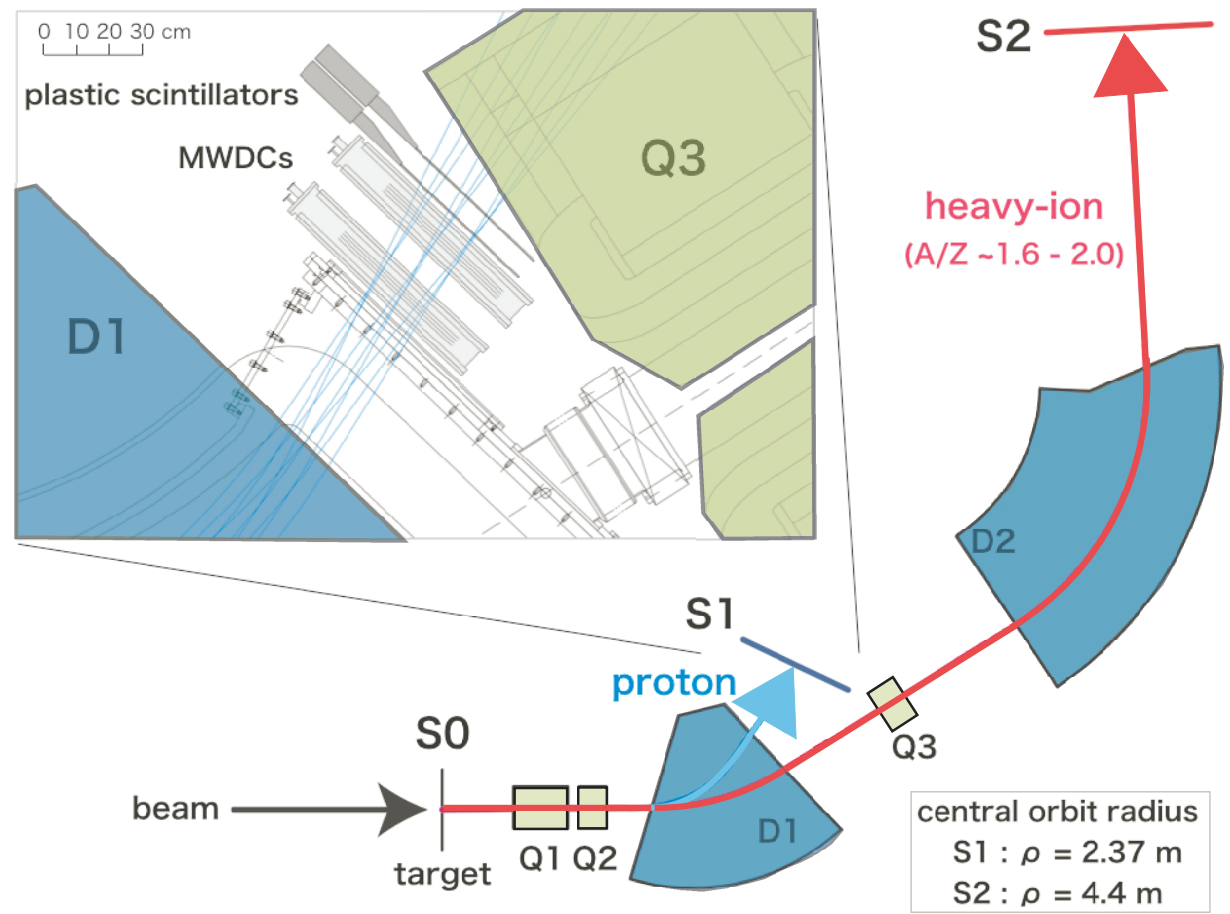

Figure 6: Schematic layout of the SHARAQ spectrometer for the separation-flow mode [ए2].

following relation:

$$
m / q=\frac{B \rho}{c} \sqrt{\left(\frac{c t}{L}\right)-1},
$$

where $m, q$ and $c$ are the nuclear mass, the charge and the light velocity respectively. $B \rho, t, L$ show magnetic rigidity, time of flight and flight length of the beam line, respectively. The diamond detectors were used to measure the time of flight, and installed at the F3 and S2 foci, which are 105 meters apart.

The experiment was performed by adopting the dispersion-matching mode [0] with full momentum acceptance $(\Delta p / p=0.6 \%)$. In this mode, the SHARAQ spectrometer and HRB were designed to satisfy the lateral and angular dispersion matching conditions. The designed momentum resolution and angular resolution of the total system are $\delta p / p=1 / 15000$ and $1 \mathrm{mrad}$, respectively, from a first-order ion-optical calculation.

The detectors installed in the beamline determined $B \rho$ and $t$ in the equation (4.]) and additionally obtained information for the ion-optical correction concerning the flight length $L$. Primarily the diamond detectors measure TOF, and the parallel-plate avalanche chamber (PPAC) at the S0 determines $B \rho$. When a multiple scattering at S0 PPAC was taken into account, the flight length $L$ was considered to attribute $B \rho$ and the beam profile at F3 and S2. Hence the $L$ was evaluated by using the tracking information at $\mathrm{F} 3, \mathrm{~S} 0$ and $\mathrm{S} 2$.

The mass spectrum corrected by those measures is shown in Fig. Z(a). The nuclei were clearly separated and identified without $\mathrm{Z}$ selection. The preliminary mass resolution for ${ }^{54} \mathrm{Ca}$ was achieved to be $m / \delta m=10500$. Although the data analysis is going on, we estimate to determine 

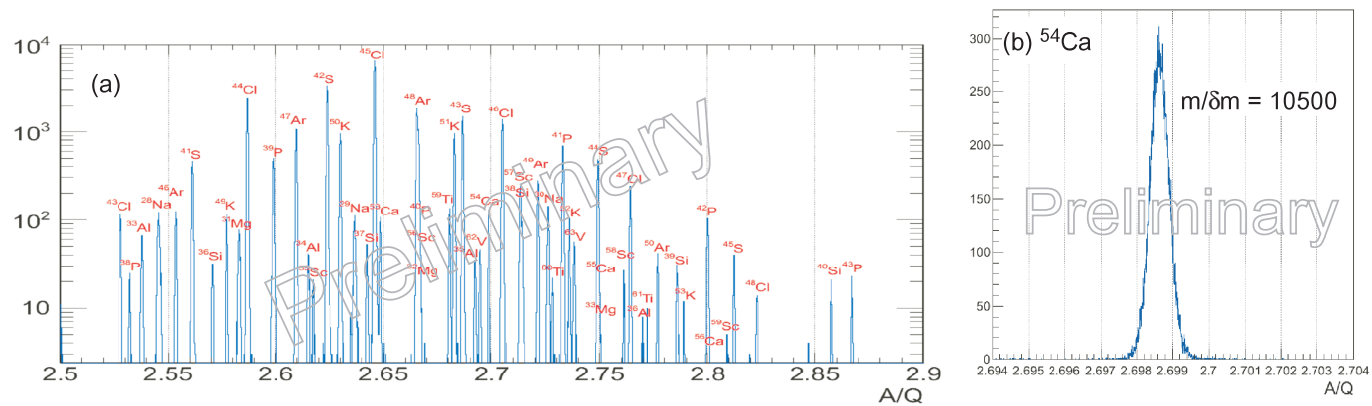

Figure 7: (a) A/Q spectrum for all nuclei measured in the present experiment. (b) The A/Q spectrum enlarged to ${ }^{54} \mathrm{Ca}$.

finally the masses of ${ }^{55} \mathrm{Ca}$ and ${ }^{56} \mathrm{Ca}$ with a mass resolution of better than $300 \mathrm{keV}(\sigma)$.

\section{Summary}

We are developing experimental technique to explore nuclear physics by take advantage of the performance of the SHARAQ spectrometer. Furthermore beamline detectors are also developed for enhancing of the performance. The LP-MWDC [ [ $]$ was developed to achieve both high rate and high position resolution, and the pCVD diamond detector [ए]] provided excellent good timing resolution.

By using these detectors, we developed the missing-mass spectroscopy of the nuclear reactions involving unbound nuclei. Recently, we have reported the results on reaction measurements of the $\left({ }^{8} \mathrm{He},{ }^{8} \mathrm{Be}\right)[\mathbb{W}]$ and $\left.\left({ }^{16} \mathrm{O},{ }^{16} \mathrm{~F}\right)[\mathbb{[}]\right]$ reactions. Since these reactions respectively have outstanding features which were not seen in reactions involving outgoing bound nuclei, we developed new capability of multi-particle detection of SHARAQ at the focal planes S2 and S1.

The direct mass measurement [5] was motivated by its physics interest, and additionally this experiment pursued the high-resolution aspect of the SHARAQ spectrometer. We successfully achieved good mass resolution beyond $m / \delta m=10000$. Therefore, in neutron-rich Ca isotopes, we will report the masses of ${ }^{55} \mathrm{Ca}$ and ${ }^{56} \mathrm{Ca}$ nuclei, and will discuss the $\mathrm{N}=34$ magic number based on the experimental result.

\section{Acknowledgments}

The authors are grateful for the continuous support from the Center for Nuclear Study, the University of Tokyo and from the RIKEN Nishina Center for Accelerator-Based Science. The present work was partly supported by a Grant-in-Aid for Scientific Research (Nos. 17002003, 19204024 and 22740150) by the Ministry of Educations, Culture, Sports, Science and Technology.

\section{References}

[1] T. Uesaka, S. Shimoura, H. Sakai, Prog. Theor. Exp. Phys. 2012 (2012) 1.

[2] S. Michimasa et al., Nucl. Instrum. Methods Phys. B 317 (2013) 305. 
[3] T. Kawabata et al., Nucl. Instrum. Methods Phys. B 266 (2008) 4201.

[4] T. Kubo et al., Nucl. Instrum. Methods Phys. B 204 (2003) 97.

[5] M. Kobayashi et al., Oral presentation in INPC 2016, Aderaide, Australlia.

[6] H. Miya et al., Nucl. Instrum. Methods Phys. B 317 (2013) 701.

[7] Y. Kiyokawa, Master thesis, The University of Tokyo, 2016.

[8] N. Fukuda et al., Nucl. Instrum. Methods Phys. B 317 (2013) 323.

[9] H. Baba et al., Nucl. Instrum. Methods Phys. A 777 (2015) 75.

[10] S. Michimasa et al., Nucl. Instrum. Methods Phys. B 317 (2013) 710.

[11] K. Kisamori et al., Phys. Rev. Lett. 116 (2016) 052501.

[12] M. Dozono et al., Nucl. Instrum. Methods Phys. A 830 (2016) 233. 